\title{
Dispersing Light with Surface Plasmon Polaritonic Crystals
}

\author{
V. Mikhailov, ${ }^{1}$ G. A. Wurtz, ${ }^{2}$ J. Elliott, ${ }^{2}$ P. Bayvel, ${ }^{1}$ and A. V. Zayats ${ }^{2, *}$ \\ ${ }^{1}$ Department of Electronic and Electrical Engineering, University College London, Torrington Place, London WC1E 7JE, \\ United Kingdom \\ ${ }^{2}$ Centre for Nanostructured Media, IRCEP, The Queen's University of Belfast, Belfast BT7 1NN, United Kingdom
}

(Received 7 March 2007; published 22 August 2007)

\begin{abstract}
Spectral dispersion of light on a finite-size surface plasmon polaritonic (SPP) crystal has been studied. The angular wavelength separation of one or more orders of magnitude higher than in other state-of-theart wavelength-splitting devices available to date has been demonstrated. The two-stage process is responsible for the dispersion value, which involves conversion of the incident light into SPP Bloch modes of a nanostructure followed by the SPP Bloch waves refraction at the SPP crystal boundary. The high spectral dispersion achievable in plasmonic devices may be useful for integrated high-resolution spectroscopy in nanophotonic, optical communication and lab-on-a-chip applications.
\end{abstract}

DOI: 10.1103/PhysRevLett.99.083901

Recent progress in photonics and integrated optics presents many challenges for development of micro- and nano-integrated components capable of routing and manipulating light waves in confined geometries. To this end, plasmonic devices offer a range of functionalities for guiding of light and its control with external signals, enhancing nonlinear interactions, and designing optical transmission and reflection properties, all with planar, subwavelength size components $[1,2]$. After development of many basic elements of two-dimensional (2D) surface plasmon polaritonic (SPP) optics [2], a search for additional functionalities in SPP-based devices continues to attract significant attention for applications in various areas of science and technology.

One of the functionalities that has not yet been addressed with plasmonic waves is dispersion and spectral analysis. Since the first experiments by Newton on light dispersion in a glass prism, the spectral dispersion of light - that is, the dependence of generalized refractive index on frequency - is ubiquitous in applications ranging from spectroscopic devices to sensing and novel optical communication technologies. Very recently, new challenges have arisen for spectroscopic devices needed in optical switching and routing of information according to its frequency (color), to develop compact devices that can be integrated into photonic circuits of the future or into labon-a-chip applications for in situ spectral functionality. These cover high-resolution spectroscopy, wavelength demultiplexing of optical signals, ultrashort pulse analysis, and many others.

Although free-space plane and concave gratings can achieve high values of spectral dispersion, their disadvantages are that they are bulky and require discrete optical components. The control of aberration is limited to a relatively narrow wavelength range, and additional components are also needed for coupling to optical fibers or waveguides [3,4]. Diffraction gratings based on planar waveguide technology are limited to a relatively small
PACS numbers: 42.79.Dj, 42.25.Fx, 78.67. $-\mathrm{n}, 78.68 .+\mathrm{m}$

size because of the difficulty of controlling the substrate quality over a large area, and they suffer from strong scattering and require complex waveguide fabrication $[4,5]$.

Recently two-dimensional spectral dispersion effects have been studied in photonic crystal slabs [6-9]. This so-called superprism effect relies on the ability to direct the light transmitted through a boundary of $2 \mathrm{D}$ photonic crystal in different directions (i.e. with large angular separation), as a function of only very small variations in light frequency. This effect arises from the very strong dispersion of photonic eigenmodes $d \vec{k} / d \omega$ (where $\vec{k}$ is the photon wave vector and $\omega$ is the light frequency) near the edges of the photonic band gap. Thus, the dispersion can be synthesized for a given wavelength range and required propagation direction. The photonic crystal superprism effect provides high spectral dispersion (up to $50^{\circ}$ deviation of the light path for the incident wavelength change of $2 \%$ ) but requires cumbersome arrangements for light coupling in or out of the 2D photonic crystal slab $[7,8]$.

In this Letter we present a new dispersive element based on a surface plasmon polaritonic crystal (SPPC) [2] which combines functionalities of a diffraction grating and the photonic crystal superprism and provides angular spectral dispersion 1 to 2 orders of magnitude higher than in the state-of-the-art planar integrated diffraction gratings and photonic crystal superprisms. We demonstrate this effect in a finite-size SPP crystal that can convert an out-of-plane (3D) multiwavelength input into an in-plane (2D) SPP output, with different frequencies propagating in different directions on a metal surface.

The proposed approach is based on the properties of surface plasmon polaritons - intrinsically 2D surface electromagnetic waves - on a periodically nanostructured metal surface forming a surface polaritonic crystal [2]. If a SPP crystal is conventionally illuminated with a light beam, SPP eigenmodes (Bloch waves), allowed to propagate on a periodic structure, are excited at certain frequen- 
cies in specific directions at which the diffraction provides the wave vector conservation:

$$
\vec{k}_{\mathrm{BSP}}=\frac{\omega}{c} n_{s} \sin \theta \vec{u}_{x y} \delta_{p} \pm p \frac{2 \pi}{D} \vec{u}_{x} \pm q \frac{2 \pi}{D} \vec{u}_{y} .
$$

Here, $k_{\mathrm{BSP}}$ is the SPP Bloch wave vector on the periodically structured surface, $\omega$ is the frequency of the illuminating light, $c$ is the speed of light, $n_{s}$ is the refractive index of the medium through which the film is illuminated, $\theta$ is the angle of incidence, $\vec{u}_{x y}$ is the unit vector in the plane of the film in the direction of the incident light wave vector projection, $\delta_{p}=1$ or 0 for $p$ - or $s$-polarized incident light (relative to the sample surface), respectively; $\vec{u}_{x}$ and $\vec{u}_{y}$ are the unit reciprocal lattice vectors of the periodic structure, $D$ is the SPPC periodicity (same in both $x$ and $y$ directions), and $p$ and $q$ are integer numbers corresponding to different directions in the SPP Brillouin zone and determining direction of SPP propagation on a surface. Near the edges of the band gap of SPP crystal (where $d \omega / d k_{\mathrm{SP}}=0$ ) [Fig. 1(d)], the SPP wave vector can undergo extremely large variations with small changes in frequency of illuminating light. Thus, the respective SPP Bloch waves will be
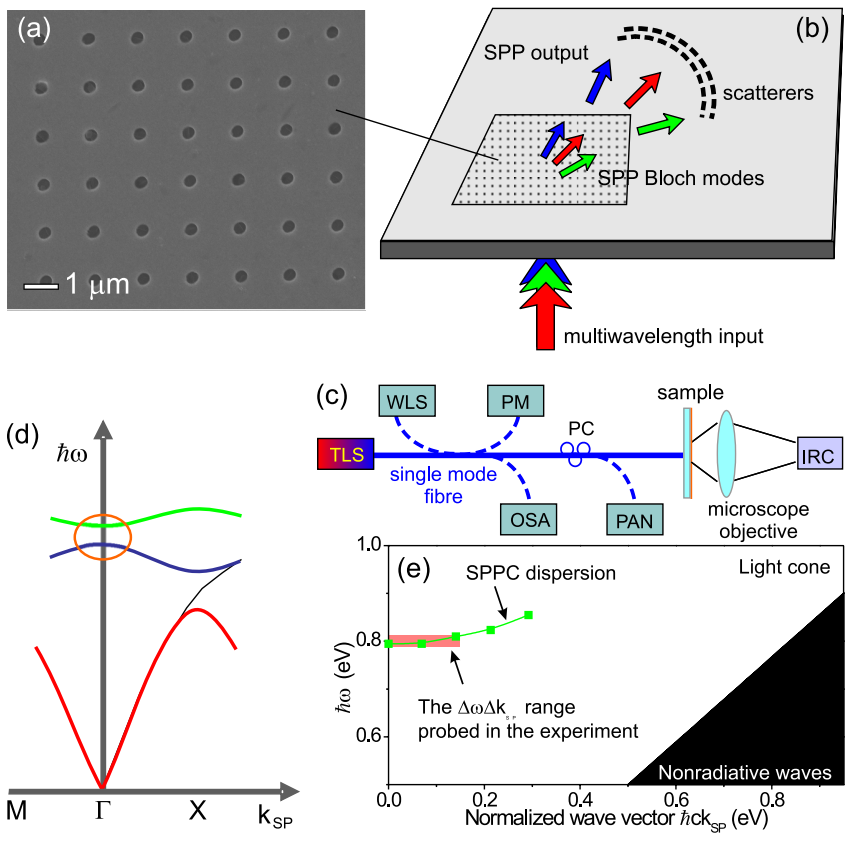

FIG. 1 (color online). (a) Electron microscope image of the SPP crystal used in the experiments; (b) Principle of the SPPC induced dispersion showing a finite-size SPPC, a set of scatterers used for SPP visualization, and illumination conditions; (c) Experimental setup: TLS - tunable laser source; WLS white light source (unpolarized); $\mathrm{PM}$ - power meter; OSAoptical spectrum analyzer; PAN — polarization analyzer; IRC infrared camera; (d) Schematics of the band structure of the SPP crystal in the vicinity of $\Gamma$ point of the Brillouin zone; the circle indicates the states accessible with normally incident Gaussian laser beam; (e) The experimentally measured SPP dispersion of the crystal shown in (a) in the range of wavelengths used in the following experiments. excited and, after crossing the boundary, SPPs will emerge from the SPP crystal onto the unpatterned part of the surface in significantly different directions [Fig. 1(b)]. Thus, SPP crystal can convert out-of-plane multiwavelength input into in-plane SPP output with different light frequencies propagating in different directions on the smooth surface. The size and shape of a nanostructure and its period can then be optimized to achieve the required SPP dispersion and even design flat SPP bands, ideal for spectral routing applications $[10,11]$.

The surface polaritonic crystals used in the present work were fabricated in thin $(50 \mathrm{~nm}) \mathrm{Au}$ films deposited onto a glass substrates [Fig. 1(a)]. Square lattice SPP crystal consisting of the arrays of circular holes were fabricated with focused ion beam milling. The periodicity of the array $(1500 \mathrm{~nm})$ and the hole diameter $(300 \mathrm{~nm})$ were chosen to achieve a band gap and, thus, high density of SPP Bloch mode states and flattening of SPP bands in the spectral range which corresponds to optical communication wavelengths around $1.5 \mu \mathrm{m}$ [Fig. 1(e)]. Near the finite-size SPP crystal $\left(50 \times 50 \mu \mathrm{m}^{2}\right.$ total area) a set of scatterers was fabricated, designed to convert SPPs to light, which is then detected in the experiments. The scatterers are located at the distance of about $40 \mu \mathrm{m}$ from the SPP crystal edge. The SPP propagation length on the Au-air surface in this wavelength range is around $200 \mu \mathrm{m}$ and sufficient to carry energy to the scatterers. The scatterer size and shape have been chosen to provide efficient coupling of the SPP waves into light beams.

For spectral dispersion measurements, the infrared (IR) light from a fiber-pigtailed tunable laser was directly coupled to the SPP crystal using a cleaved single mode fiber. The polarization was controlled using an all-fiber polarization controller and the output was monitored with a polarimeter [Fig. 1(c)]. In this way Gaussian beam illumination of the SPP crystal was obtained with the wave vector components in the range of incident angles $0^{\circ}-10^{\circ}$. Illumination spot of about $10 \mu \mathrm{m}$ diameter was positioned at the center of the array. As described above, the input light excites SPP modes that are then scattered by nearby surface scatterer, and the resultant scattered light imaged with a high-sensitivity IR camera. The images recorded at different wavelengths and polarizations of the illuminating light were used to visualize the dispersive properties of the structures.

The images, showing the illuminated SPP crystal with the surrounding scatterers and the neighboring second SPP crystal, are presented in Fig. 2. The SPPs are excited on the illuminated (right-hand side of Fig. 2) SPP crystal and after traversing the SPP crystal boundary propagate on the smooth surface. Only the SPP waves excited along the normal to the facet of the illuminated SPP crystal in the horizontal direction can reach the second crystal (left-hand side of Fig. 2) situated in the far-field of the illuminated one. These SPP waves are then scattered in light. Because of the longitudinal nature of the SPP field, in order to be coupled to SPP wave, the incident light must have its 

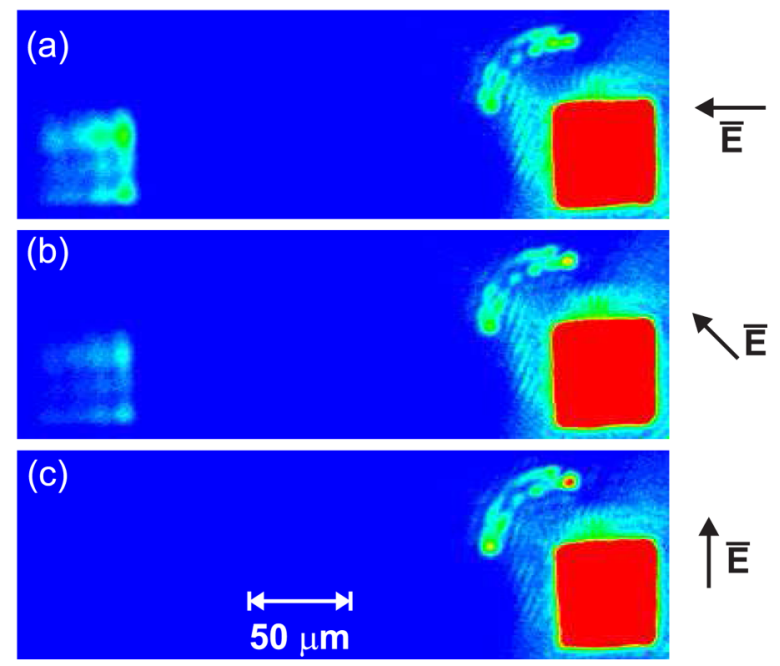

FIG. 2 (color online). Series of images of the intensity distributions over the metal surface for the different polarizations of the incident light as indicated by the arrows. The illuminating light wavelength is $\lambda=1523 \mathrm{~nm}$.

electric field vector perpendicular to the surface or parallel to the SPP propagation direction [2]. When the polarization of the excitation is changed, SPPs are excited in a different direction (in the direction of the electric field for the case of normal incidence) and the intensity over the probing SPP crystal changes. For the polarization orthogonal to the direction between the two arrays, the SPPs are not excited toward the probing array and, therefore, not observed in the optical images. This polarization behavior (Fig. 2) confirms the nature of the contributing surface waves. However, the SPP waves propagating along the normal to the SPP crystal facet are not of interest for spectroscopic applications, since their wave vector is not conserved when they refract on the SPPC boundary $[9,12]$.

For the investigations of the spectral dispersion we have chosen a set of scatterers that are not illuminated directly by the normal SPP wave, thus reducing wavelengthindependent background illumination. The polarization behavior of the SPP intensity reaching these scatterers is more complex since the various diffraction orders are not fully separated, but in general follows the polarization dependence of the SPP wave excitation.

The images of the light distributions at the scatterers reveal strong frequency dependence of the SPP intensity around the crystal (Fig. 3). The intensity of SPPs reaching the scatterers situated around the semicircle varies with the light frequency and polarization: as the frequency of the incident light changes, the scatterers situated at different angles with respect to the crystal are illuminated by the SPP beams differently, since the light of different wavelengths is coupled to SPP waves propagating in different directions on a smooth metal surface. The images measured with unpolarized white light illumination show uniform distribution of light intensity around the scatterers apart from D2 (Fig. 3), where the intensity is significantly
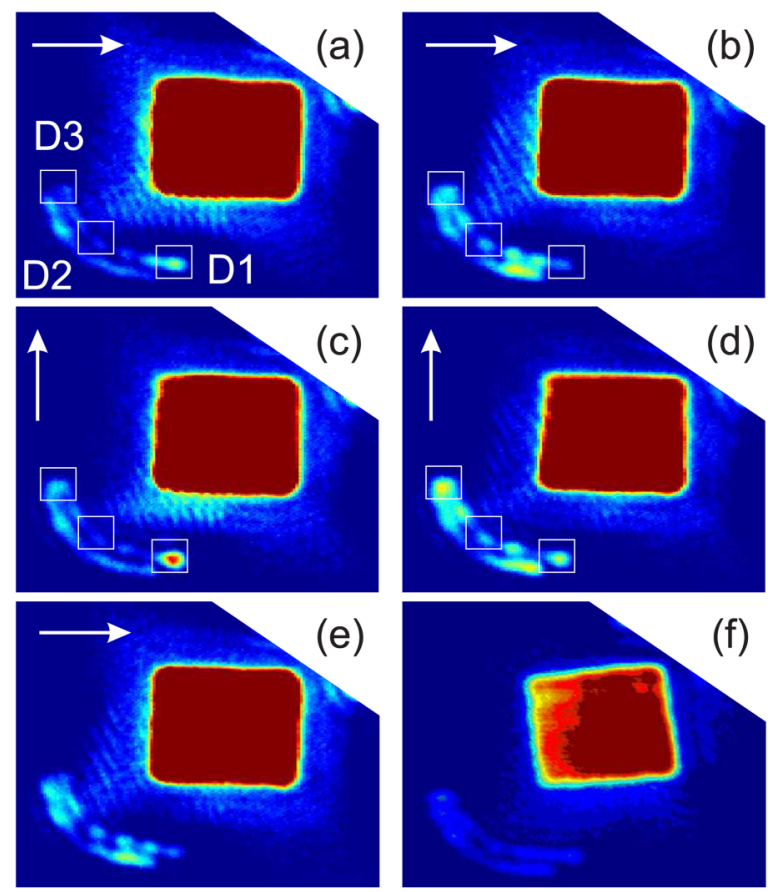

FIG. 3 (color online). Series of images of the intensity distributions over the metal surface for the different wavelengths and polarizations of the incident light: (a), (c) $\lambda=1524.4 \mathrm{~nm}$, (b), (d) $\lambda=1524.8 \mathrm{~nm}$, (e) $\lambda=1552.0 \mathrm{~nm}$ (incident light polarization is indicated by arrows); (f) unpolarized white light. Some of the representative scatterers are indicated by the squares $D$ on the images.

lower as this scatterer is the closest to the corner of the SPP crystal. Lower intensity is always observed at D2 in spectral measurements (Figs. 3 and 4). The finite range of angles of incidence obtained with the Gaussian beam illumination may result in cross talk between different wavelengths.

The wavelength dependencies of the light scattered by D1 and D2 (as marked in Fig. 3) are shown in Fig. 4(a) and
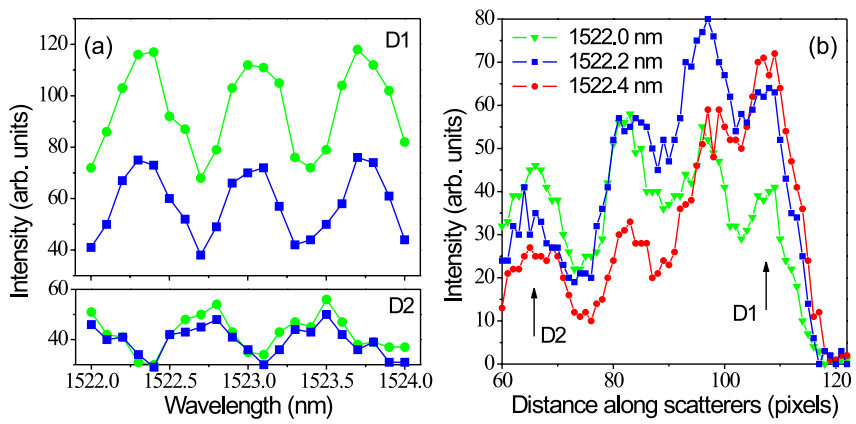

FIG. 4 (color online). (a) The wavelength dependencies of the intensity averaged over the scatterers D1 and D2 marked on the images in Fig. 3 for vertical (circles) and horizontal (squares) polarizations of the incident light as indicated in Fig. 3. (b) Cross sections along the four scatterers between the scatterers D1 and D2 for different wavelengths of the incident light. Please note that due to nonlinearity of the imaging camera response, the contrast may be suppressed on the presented plots. 
reveal strong changes of the scattering intensity with small changes of the frequency of light. Similarly, the cross sections taken along the set of scatterers for different frequencies confirm the intensity changes at the scatterers for very small values of frequency variation. This shows the extremely high dispersion of the surface polaritonic crystal, which is estimated from the measured images to be about $(20-30)^{\circ} / \mathrm{nm}$ in this spectral range [13]. This value is an order of magnitude higher than the values $(1-2)^{\circ} / \mathrm{nm}$ reported with photonic crystal superprisms $[7,8]$ and 2 orders of magnitude higher than those observed with conventional planar diffraction gratings $[3,14]$.

Such strong spectral dispersion is achieved due to the two-stage process involving SPP Bloch waves on a periodically structured surface. Firstly, the illumination of the SPP crystal by light leads, due to diffraction on a periodic structure, to excitation of SPP Bloch waves with different frequencies of the incident light being coupled to the SPP Bloch modes propagating in different directions. This first stage provides the angular dispersion, which can be estimated from the conditions of the excitation of the SPP waves on a smooth surface by a periodic grating to be about $0.1^{\circ} / \mathrm{nm}$ in the operating spectral range. (Please note that the above value is similar to those obtained with conventional diffraction gratings, reflecting the fact that it is related to a conventional diffraction process.) In the case of the SPP Bloch modes in the SPP crystal this value can only be higher, as discussed above, due to the flattening of the allowed bands of the crystal. The second stage that enhances the dispersion is the diffraction of the SPP Bloch mode on the periodic structure formed by the termination of the SPP crystal. This two-dimensional process can be considered as that of refraction on the SPP crystal boundary: the wave vector component parallel to the boundary must be conserved for the Bloch wave inside the crystal and the SPP wave outside it $[9,12]$. This is analogous to a 2D photonic crystal superprism with both positive and negative refraction allowed $[9,15]$. The reported values for the measured superprism dispersion in $2 \mathrm{D}$ photonic crystals are consistent with our measured values for this process estimated from the total angular dispersion of the two-stage process studied. Because of a finite range of the excitation wave vectors, the overall intensity at the scatterers may be determined by a combination of various SPP modes refracted on the SPP crystal boundary, similar to decomposition of Bloch waves on a photonic crystal boundary [16].

In conclusion, we have proposed a new principle of highly dispersive elements based on SPP crystals. These can convert an out-of-plane multiwavelength input signal into an in-plane SPP output on a metal surface, with different light frequencies spatially separated. The high dispersion values are the result of a two-stage process relying on the conversion of the incident light into SPP Bloch waves on a SPP crystal and, then, Bloch waves traversing the SPP crystal boundary. High spectral dispersion and ease of use make the SPPC-based dispersive elements very promising for nanophotonic applications where wavelength splitting and separation is needed - it can be achieved in a compact integrated device which can be incorporated into lab-on-achip or other photonic integrated circuits such as demultiplexers, routers, or switches for optical communications. Moreover, thanks to surface plasmon properties, this dispersion can be controlled optically or electrically using active metallodielectric nanostructures [2,17].

The financial support from EPSRC is gratefully acknowledged. The authors thank W. Dickson and R. Pollard for help with the sample fabrication and V. Tsatourian for help with computer programming.

*a.zayats@qub.ac.uk.

[1] W. L. Barnes, A. Dereux, and T.W. Ebbesen, Nature (London) 424, 824 (2003).

[2] A. V. Zayats, I. I. Smolyaninov, and A. A. Maradudin, Phys. Rep. 408, 131 (2005).

[3] I. V. Peisakhson, J. Opt. Technol. 69, 17 (2002).

[4] Y. V. Bazhanov et al., J. Opt. Technol. 73, 445 (2006).

[5] E. G. Churin and P. Bayvel, Electron. Lett. 34, 1225 (1998).

[6] H. Kosaka et al., Phys. Rev. B 58, R10096 (1998); L. Wu, M. Mazilu, T. Karle, and T. F. Krauss, IEEE J. Quantum Electron. 38, 915 (2002).

[7] T. Baba and M. Nakamura, IEEE J. Quantum Electron. 38, 909 (2002).

[8] J. J. Baumberg et al., Appl. Phys. Lett. 85, 354 (2004).

[9] C. Luo, S. G. Johnson, J.D. Joannopoulos, and J. B. Pendry, Phys. Rev. B 65, 201104(R) (2002).

[10] M. Kretschmann and A. A. Maradudin, Phys. Rev. B 66, 245408 (2002).

[11] I. R. Hooper and J. R. Sambles, Phys. Rev. B 65, 165432 (2002).

[12] W. Jiang, R. T. Chen, and X. Lu, Phys. Rev. B 71, 245115 (2005).

[13] The series of images of the intensity distributions over the scatterers were recorded with the wavelength step of $0.1 \mathrm{~nm}$. Knowing the distance between the neighboring scatterers and the wavelengths at which the intensity maxima are achieved on the scatterers, the dispersion was evaluated.

[14] K. Oya et al., Opt. Lett. 30, 192 (2005).

[15] I. I. Smolyaninov, C. C. Davis, J. Elliott, G. A. Wurtz, and A. V. Zayats, Phys. Rev. B 72, 085442 (2005).

[16] E. Cassan, D. Bernier, G. Maire, D. Marris-Morini, and L. Vivien, J. Opt. Soc. Am. B 24, 1211 (2007).

[17] G. A. Wurtz, R. Pollard, and A. V. Zayats, Phys. Rev. Lett. 97, 057402 (2006). 